Research

Open Access

\title{
A randomized trial of chlorhexidine gluconate on oral bacterial pathogens in mechanically ventilated patients
}

\author{
Frank A Scannapieco1, Jihnhee $\mathrm{Yu}^{2}$, Krishnan Raghavendran ${ }^{3,4,7}$, Angela Vacanti ${ }^{1}$, \\ Susan I Owens ${ }^{5}$, Kenneth Wood 5 and Joseph M Mylotte ${ }^{6}$
}

\author{
1Department of Oral Biology, School of Dental Medicine, University at Buffalo, The State University of New York, 3435 Main St. Buffalo, NY 14214, \\ USA \\ 2Department of Biostatistics, School of Public Health and Health Professions, The State University of New York, 3435 Main Street, Buffalo, NY \\ 14214, USA \\ ${ }^{3}$ Department of Surgery and Anesthesiology, School of Medicine and Biomedical Sciences, The State University of New York, 3435 Main Street, \\ Buffalo, NY 14214, USA \\ 4Erie County Medical Center, 462 Grider Street, Buffalo, NY 14215, USA \\ ${ }^{5}$ Frontier Science and Technology Research Foundation, 4033 Maple Road, Amherst, NY 14266, USA \\ ${ }^{6}$ Department of Medicine, School of Medicine and Biomedical Sciences, The State University of New York, 3435 Main Street, Buffalo, NY 14214, \\ USA \\ ${ }^{7}$ Current address: Department of Surgery, University of Michigan, 1C421 University Hospital, SPC 50331500 E. Medical Center Drive, Ann Arbor, \\ MI 48109, USA
}

Corresponding author: Frank A Scannapieco, fas1@buffalo.edu

Received: 20 Feb 2009 Revisions requested: 26 Mar 2009 Revisions received: 23 Jun 2009 Accepted: 15 Jul 2009 Published: 15 Jul 2009

Critical Care 2009, 13:R117 (doi:10.1186/cc7967)

This article is online at: http://ccforum.com/content/13/4/R117

(C) 2009 Scannapieco et al.; licensee BioMed Central Ltd.

This is an open access article distributed under the terms of the Creative Commons Attribution License (http://creativecommons.org/licenses/by/2.0), which permits unrestricted use, distribution, and reproduction in any medium, provided the original work is properly cited.

\begin{abstract}
Introduction Dental plaque biofilms are colonized by respiratory pathogens in mechanically-ventilated intensive care unit patients. Thus, improvements in oral hygiene in these patients may prevent ventilator-associated pneumonia. The goal of this study was to determine the minimum frequency (once or twice a day) for $0.12 \%$ chlorhexidine gluconate application necessary to reduce oral colonization by pathogens in 175 intubated patients in a trauma intensive care unit.

Methods A randomized, double-blind, placebo-controlled clinical trial tested oral topical $0.12 \%$ chlorhexidine gluconate or placebo (vehicle alone), applied once or twice a day by staff nurses. Quantitation of colonization of the oral cavity by respiratory pathogens (teeth/denture/buccal mucosa) was measured.
\end{abstract}

Results Subjects were recruited from 1 March, 2004 until 30 November, 2007. While 175 subjects were randomized, microbiologic baseline data was available for 146 subjects, with 115 subjects having full outcome assessment after at least 48 hours. Chlorhexidine reduced the number of Staphylococcus aureus, but not the total number of enterics, Pseudomonas or Acinetobacter in the dental plaque of test subjects. A nonsignificant reduction in pneumonia rate was noted in groups treated with chlorhexidine compared with the placebo group (OR $=0.54,95 \% \mathrm{Cl}: 0.23$ to $1.25, P=0.15$ ). No evidence for resistance to chlorhexidine was noted, and no adverse events were observed. No differences were noted in microbiologic or clinical outcomes between treatment arms.

Conclusions While decontamination of the oral cavity with chlorhexidine did not reduce the total number of potential respiratory pathogens, it did reduce the number of $S$. aureus in dental plaque of trauma intensive care patients.

Trial Registration clinicaltrials.gov NCT00123123.

ANOVA: analysis of variance; APACHE: Acute Physiology and Chronic Health Evaluation; BqBAL: blind quantitative bronchoalveolar lavage; cfu: colony forming units; $\mathrm{CHX}$ : chlorhexidine gluconate; Cl: confidence interval; CPIS: clinical pulmonary infection score; ECMC: Erie County Medical Center; $\mathrm{FiO}_{2}$ : fraction of inspired oxygen; $\mathrm{HR}$ : hazards ratio; MV-ICU: mechanically ventilated, intensive care unit; OR: odds ratio; $\mathrm{PaO}$ : partial pressure of arterial oxygen; PRPs: potential respiratory bacterial pathogens; SID: subject Identification Numbers; VAP: ventilator-associated pneumonia. 


\section{Introduction}

The pathogenesis of ventilator-associated pneumonia (VAP) involves aspiration of bacteria from the oropharynx into the lung, and subsequent failure of host defenses to clear the bacteria resulting in development of lung infection [1]. In mechanically ventilated, intensive care unit (MV-ICU) patients, the major potential respiratory bacterial pathogens (PRPs) include Staphylococcus aureus, Pseudomonas aeruginosa, Acinetobacter species, and enteric species. Previous studies have shown that dental plaque and the oral mucosa are often colonized by PRPs [2,3]. Also, PRPs from dental plaque of MV-ICU patients are genetically identical to strains from bronchoscopic cultures taken at the time pneumonia was suspected, $[4,5]$. These findings suggest that dental plaque may be an important reservoir of PRPs that cause VAP. Thus, improving oral hygiene in MV-ICU patients and reducing dental plaque load on teeth has the potential to reduce the risk of VAP.

Topical oral application of antiseptics such as chlorhexidine gluconate $(\mathrm{CHX})$ have been evaluated for the prevention of VAP. $\mathrm{CHX}$ is a cationic chlorophenyl bis-biguanide antiseptic that has long been approved for use as an inhibitor of dental plaque formation and gingivitis [6-8]. $\mathrm{CHX}$ is of particular interest as an oral disinfectant in MV-ICU patients because of its substantivity (the ability of $\mathrm{CHX}$ to bind to oral tissues with subsequent slow release and thus a relatively long period of action). Several recently published clinical trials of intra-oral disinfection with topical $\mathrm{CHX}$ [9-15] or povidone-iodine gargle and tooth-brushing [16] have demonstrated a reduction in the prevalence of oropharyngeal colonization by PRPs, as well as a reduction in the rate of VAP in MV-ICU patients. Based on these observations, recommendations for preventing VAP have included improving oral hygiene in MV-ICU patients $[17,18]$. However, not all studies of the use of $\mathrm{CHX}$ have shown a reduction in the incidence of pneumonia [19]. Moreover, the studies that have been published used different $\mathrm{CHX}$ dosing regimes and did not always clearly define the method of application of $\mathrm{CHX}$. Identification of the minimum frequency for $\mathrm{CHX}$ application required to reduce the number of PRPs on the teeth may promote the routine use of this intervention for MV-ICU patients. However, the most simple method for application and the minimally effective dosing regime of $\mathrm{CHX}$ that improves oral hygiene to reduce the number of PRPs in dental plaque biofilms has not been determined.

Thus, the goal of the present study was to determine the minimal frequency of oral $\mathrm{CHX}$ application (once or twice a day), compared with placebo, which significantly reduces oral colonization by PRPs in MV-ICU patients. Secondary endpoints included incident VAP, duration of mechanical ventilation, length of ICU stay, and rate of mortality.

\section{Materials and methods Patient population}

This trial was approved by the University at Buffalo Institutional Review Board. Subjects for this study were recruited from patients admitted to the 18-bed trauma ICU of the Erie County Medical Center (ECMC) who were mechanically ventilated. ECMC is a 550-bed inpatient level 1 regional center for trauma, burn care, and rehabilitation and is an affiliated teaching facility for the State University of New York at Buffalo. This ICU is 'closed', in that all patients who are admitted are primarily managed by the study intensivist physician (KR) or his surrogates. The average length of stay in this unit in the year before the start of the study was approximately six days. Over the past five years the incidence of VAP has ranged from 10 to $50 \%$ of all MV patients, and VAP per 1000 ventilator days has ranged from 8 to 12 . Once participant eligibility was established, written informed consent was obtained from each patient, or most often from his/her next of kin or health care proxy.

\section{Sample size estimate}

Based on previous studies, it was conservatively estimated that approximately $50 \%$ of all subjects admitted to the ICU would be colonized by a PRP. In order to have a $90 \%$ power of detecting a difference between colonization proportions, and assuming a dose-response effect whereby $50 \%$ of the placebo group would be presumed to be colonized, with $25 \%$ of the twice daily $\mathrm{CHX}$ group, and $20 \%$ of the once daily $\mathrm{CHX}$ group, it was determined that a minimum group size would require 53 participants per treatment arm.

\section{Inclusion/exclusion criteria}

Eligible patients were those admitted to the ICU who were expected to be intubated and mechanically ventilated within 48 hours of admission, with the exception of those demonstrating the following exclusion criteria: a witnessed aspiration (to eliminate patients with chemical pneumonitis); a confirmed diagnosis of post-obstructive pneumonia (e.g. advanced lung cancer); a known hypersensitivity to $\mathrm{CHX}$; absence of consent; a diagnosed thrombocytopenia (platelet count less than 40 and/or a INR above 2, or other coagulopathy); a do not intubate order; children under the age of 18 years; pregnant women; legal incarceration; transfer from another ICU; oral mucositis; immunosuppression (either-HIV or drug induced (e.g. organ transplant patients or those on long term steroid therapy)); and re-admission to the ICU.

\section{Trial design}

Eligible patients, after giving informed consent and following baseline assessment, were randomly assigned to one of three arms (Figure 1): 1) a control arm in which patients received twice daily oral topical applications (AM and PM) with the $\mathrm{CHX}$ vehicle control alone (having the same color, taste, and smell as the $\mathrm{CHX}$ rinse); 2) an experimental arm in which patients received once daily oral topical treatment with $0.12 \%$ 


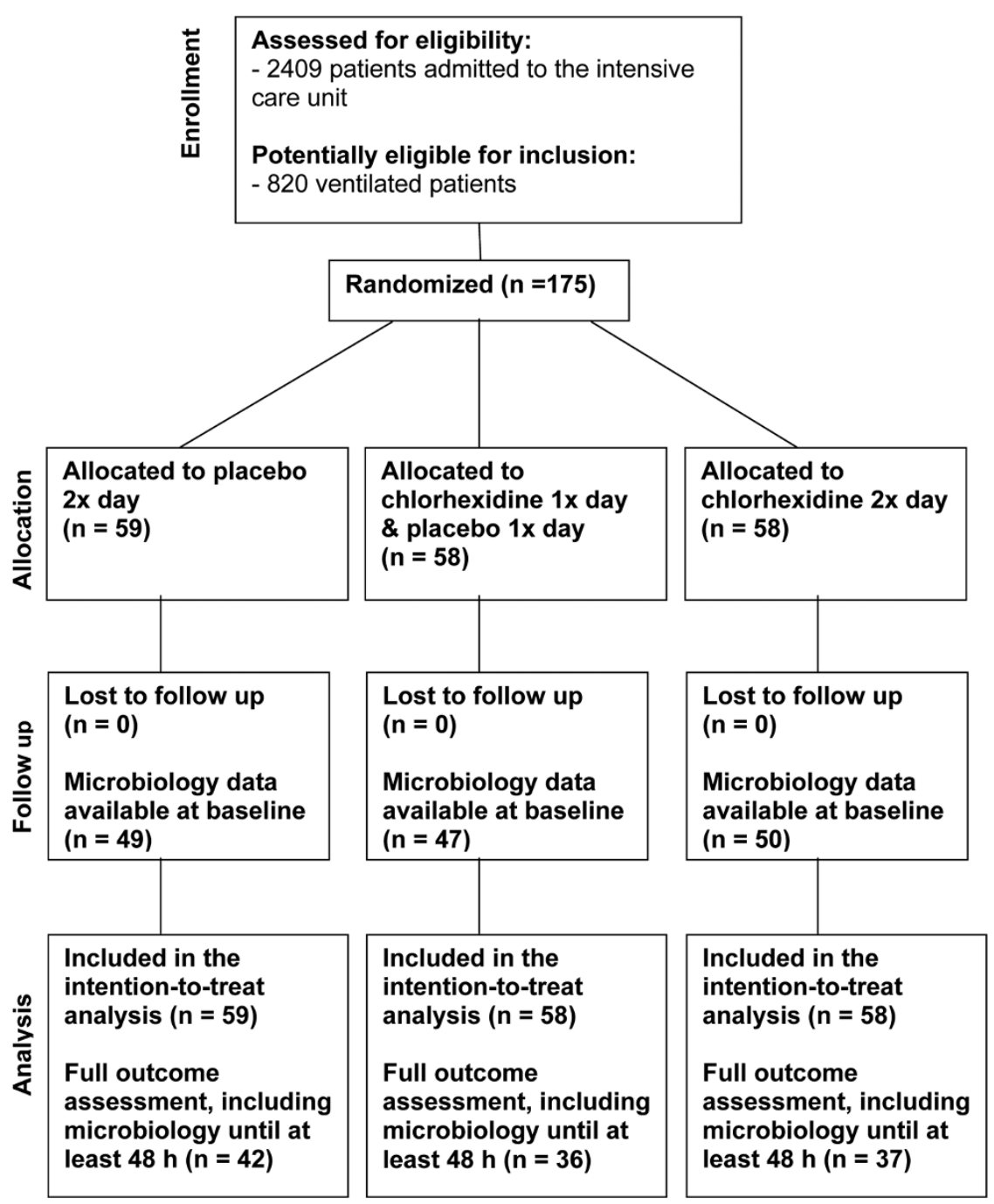

Study design. The final analysis was based on subjects having a microbiologic assessment at baseline and for at least the 48 hour ( $\mathrm{h}$ ) time point ( $\mathrm{n}$ $=42$ in the placebo group, 36 in the chlorhexidine gluconate (CHX) once a day group, and 37 in the CHX twice a day group).

$\mathrm{CHX}$ and once daily oral topical treatment with vehicle control (subjects were randomly selected to receive $\mathrm{CHX}$ in the morning and placebo in the evening, or $\mathrm{CHX}$ in the evening and placebo in the morning); and 3) an additional experimental arm in which patients received twice daily oral topical treatments with $0.12 \% \mathrm{CHX}$ gluconate.

\section{CHX formulation}

The oral rinse was formulated [20] as follows: $3 \mathrm{ml}$ chlorhexidine gluconate $20 \%$ added to $200 \mathrm{ml}$ of distilled water; separately $5 \mathrm{ml}$ essence of peppermint was mixed with $5 \mathrm{ml} 95 \%$ ethanol, and then $15 \mathrm{ml}$ glycerin. The solutions were then mixed and brought to $500 \mathrm{ml}$ with distilled water. The placebo contained all ingredients, except for $\mathrm{CHX}$, which was substituted with distilled water.

\section{Concealment, blocking, and randomization}

Subjects were randomized to the study via a web-based subject enrollment system that used protocol-specific specification files that presented questions to site personnel to evaluate eligibility. Only subjects who met all eligibility requirements were randomized to the study. The randomization system prepared a set of Subject Identification Numbers (SID) that identified individual treatment assignments. The study nurse obtained the SID number based on the randomization when the subject was enrolled. The assigned SID number was sent to the study pharmacist to dispense the appropriate blinded treatment. Assignment of treatment was blinded to patients and all investigators including outcome assessors, statisticians, and care providers. Sealed envelopes containing a random number were generated in blocks of six to provide 
concealment of patient assignment from the investigators. Each block of six numbers were assigned corresponding boxes prepared and numbered sequentially from one to six. The pharmacists prepared each box to contain two 32 oz bottles, one labeled AM and one labeled PM. For each block one of 720 possible permutations of symbols (Placebo $1\left(\mathrm{P}_{1}\right)$, Placebo $2\left(\mathrm{P}_{2}\right)$, Once AM $1\left(\mathrm{OM}_{1}\right)$, Once PM $1\left(\mathrm{OM}_{2}\right)$, Twice a day $1\left(T_{1}\right)$, Twice a day $2\left(T_{1}\right)$ ) were selected using a random number generator, and numbers one to six were assigned based on randomly selected permutations. The oral topical treatment for each box was formulated and prepared by the hospital pharmacy.

\section{Intervention}

$\mathrm{CHX}$ was applied using a rinse-saturated oral foam applicator (Sage Products, Cary, IL, USA) twice each day (in the morning at about $8 \mathrm{AM}$ and in the evening at about $8 \mathrm{PM})$. Applications were for one minute with about $1 \mathrm{oz}$ of $0.12 \% \mathrm{CHX}$ or placebo. The ICU staff nurses performed $\mathrm{CHX}$ application. All teeth, the oral soft tissues including buccal mucosa, vestibule, gingiva, and the floor of the mouth and tongue dorsum were swabbed. Excess rinse was suctioned out of the subject's mouth after one minute. In addition to this rinse, the routine oral care regime used by the ICU was maintained throughout the course of the trial. Briefly, routine oral care included use of a suction toothbrush (Sage Products, Cary, IL, USA) twice a day and as needed to brush teeth and the surface of the tongue, for approximately one to two minutes, and applying suction at completion and as needed during the brushing. In addition, swabbing with Peroxamint solution (Sage Products, Cary, IL, USA) was performed every four hours. This was followed by the application of a mouth moisturizer (Sage Products, Cary, IL, USA) to the oral mucosa using a fresh swab. Also, deep suctioning was performed to assist in removing oropharyngeal secretions pooled on top of the cuff of the endotracheal tube every 12 hours and following position changes.

The study nurses trained all ICU staff nurses to perform the standardized technique for application of the rinse. The study nurses periodically observed ICU staff nurses for adherence to study protocol. Routine in-service sessions were held every three months to review the protocol with ICU staff nurses. The study nurses collected all samples and study data and followed each enrolled subject until they were exited from the study.

\section{Dental plaque and airway sampling}

Dental plaque samples were collected on the day of admission to the ICU (baseline sampling) and every 48 hours thereafter until discharge from the study. Samples consisted of supragingival dental plaque pooled from at least two remaining teeth, or if the patient was edentulous, from the buccal mucosa, or, if they were wearing a denture, from the tissue surface of the maxillary denture. Samples were collected with a sterile stainless steel curette and dispersed into $2 \mathrm{ml}$ of sterile normal saline. For patients who were edentulous and not wearing dentures, the buccal mucosa was sampled by vigorous rubbing with a sterile swab and dispersed into sterile normal saline $(2 \mathrm{ml})$. Sampling was performed prior to routine daily oral care. Samples were immediately sent to the laboratory for processing.

Tracheal samples were obtained from all intubated patients at each sampling interval (baseline and every 48 hours) using a sterile suction catheter placed into the endotracheal tube. When pneumonia was suspected (see below), lung secretions were obtained and analyzed for bacterial infection by Blind Quantitative Bronchoalveolar Lavage (bqBAL) using a miniBAL technique.

\section{Microbiological procedures}

Samples were vortexed for one minute and split into 4 to 0.5 $\mathrm{ml}$ portions (with three of the samples placed at $-70^{\circ} \mathrm{C}$ ). The remaining dental plaque samples were diluted and plated using a spiral plater on sheep's blood agar (to isolate $S$. aureus), and MacConkey agar (for isolation of Gram-negative bacilli). For tracheal secretions, semi-quantitation of PRPs was performed ( 0 to $4+$ range scored). Microbial cultures were incubated for 72 hours at $37^{\circ} \mathrm{C}$ in $5 \%$ carbon dioxide. Plates were assessed for growth of the following target PRPs: $S$. aureus, $P$. aeruginosa, Acinetobacter species, and enteric organisms (Klebsiella pneumoniae, Serratia marcescens, Enterobacter species, Proteus mirabilis, Escherichia coli). Results of quantitative cultures were expressed as colony forming units (cfu) per ml. A bqBAL sample was considered positive if $10^{4} \mathrm{CFU} / \mathrm{ml}$ or more of a PRP was found.

For measurement of $\mathrm{CHX}$ resistance, dental plaque and lower airway secretions processed for microbiologic analysis as described above, were plated on trypticase soy agar with and without a final concentration of $0.12 \% \mathrm{CHX}$ gluconate. Plates were incubated both aerobically and anaerobically for 72 hours and the proportion of $\mathrm{CHX}$-resistant colonies enumerated.

\section{Patient data, outcome variables, and potential confounding variables}

All subjects entered into the study were followed for up to 21 days, or on discharge from the ICU, extubation, or death. Although it was uncertain when planning the trial that enough subjects could be recruited to discern a statistically significant effect of the oral intervention on incident VAP, we selected as primary outcome variables dental plaque score and colonization of the oral cavity by respiratory pathogens ( $S$. aureus, $P$. aeruginosa, Acinetobacter species, and the enteric species $K$. pneumoniae, S. marcescens, Escherichia species, P. mirabilis, and $E$. coli) by quantitative culture measured at baseline and subsequently at 48-hour intervals until the subject was discharged from the ICU. 
Secondary outcomes assessed were: diagnosis of pneumonia in a MV patient at least 48 hours following admission to the ICU (see below); mortality following admission and during the stay in the ICU; length of ventilation in the ICU; and length of stay in the ICU.

The clinical pulmonary infection score system (CPIS) used is based on five different elements: partial pressure of arterial oxygen $\left(\mathrm{PaO}_{2}\right)$ /fraction of inspired oxygen $\left(\mathrm{FiO}_{2}\right)$; Infiltrate on chest radiograph; Leukocytosis; purulent secretions; and fever. The CPIS score was calculated as follows: 1) Fever: 0 (36.5 to $38.4^{\circ} \mathrm{C}$ ), 1 (38.5 to 39), 2 (<36.0 OR >39.0); 2) Leukocytosis: 0 (4000 to 11,000 wh.ite blood cells per $\mathrm{mm}^{3}$ of blood), 1 (11,000 to 17,000), 2 (>17,000); 3) New infiltrate: $0=$ None, $1=$ Patchy, $2=$ Localized; 4) Secretions: $0=$ None to minimal, $1=$ moderate, $2=$ large amount; and 5) $\mathrm{PaO}_{2} /$ $\mathrm{FiO}_{2}: 0=$ more than 330 and $2=$ less than 330 .

A CPIS score 6 or more triggered sampling of the lower airway by bqBAL using a Combicath ${ }^{\circledR}$ (KOL Bio Medical Instruments, Chantilly, VA, USA). Briefly, this technique involves insertion of the Combicath through the endotracheal tube and its advancement until resistance was encountered [21,22]. The catheter was then withdrawn by a cm, the inner portion of the co-axial catheter advanced to dislodge the poly-ethylene glycol plug, and then $50 \mathrm{ml}$ of normal saline was instilled through the catheter. After 30 seconds, the specimen was withdrawn and sent for quantitative bacteriology. The presence of $10^{4}$ $\mathrm{cfu} / \mathrm{ml}$ or more of a target PRP in bqBAL fluid or a positive pleural fluid culture in the absence of previous pleural instrumentation was considered as positive evidence for a diagnosis of pneumonia.

The following demographic information was recorded: age; gender; race; admission diagnosis; admitted from i) community, ii) hospital ward, iii) other ICU, or iv) nursing home.

The following variables were assessed following patient discharge: total days of hospital admission; type of antibiotic therapy (within 14 days), and total duration in days prior to admission to the ICU.

The severity of illness score utilizing the Acute Physiology and Chronic Health Evaluation (APACHE) II system was determined for each subject at baseline [23]. This index utilizes information obtainable from the patient's hospital record, including physiologic information (temperature, mean arterial pressure, heart prevalence, respiratory prevalence, oxygenation, arterial $\mathrm{pH}$, serum levels of $\mathrm{Na}, \mathrm{K}$ and creatinine, hematocrit, white blood count), and age.

\section{Oral examination}

The study nurse performed a baseline oral examination. Follow-up oral examination and clinical sample collection was performed every 48 hours until the patient was discharged from the study. The oral examination consisted of a simplified plaque index and the enumeration of missing teeth. The plaque index was performed for six teeth (the upper right first molar, upper right central incisor, upper left first bicuspid, lower left first molar, lower left central incisor, and lower right first bicuspid). A Michigan O-probe and oral mirror were used to assess plaque. Only the mesial-buccal and mesial-lingual surfaces at the gingival margin of the teeth were scored. The amount of plaque present on the tooth was scored 0 to 3 as follows: $0=$ no plaque noted; 1 = plaque seen only on the tip of an explorer passed over the tooth surface; 2 = plaque obvious with the naked eye; $3=$ gross deposits of plaque present over the entire tooth.

The plaque index was calculated as the average of the scores obtained from the six teeth. For those patients missing any of the index teeth, or those with endotracheal tube placement and/or security that precluded examination, teeth available to be examined closest to the missing teeth were scored. Only a single score was given for each tooth, representing the surface harboring the most plaque.

The hard-palate, soft palate, buccal mucosa, tongue, and gingiva were examined for abnormalities, including inflammation, ulceration or other signs of inflammatory irritation that might be expected to be secondary to exposure to $\mathrm{CHX}$.

\section{Adverse events}

All subjects were monitored for potential adverse events, which included intraoral events (mucositis, thrush, tooth staining, alterations in taste, tooth hypersensitivity) and systemic adverse events (mortality).

\section{Data management}

Once subjects were enrolled to the study site, study personnel completed protocol specific case report forms according to the study evaluation schedule. The case report forms were entered into the study database via a distributed data entry system.

Computerized data checks were run on the data as it was entered into the database. These computerized checks were tracked for resolution. The study data manager also ran manual QA checks for inconsistent data. Inconsistent data items were queried by the study data manager, which were followed to resolution.

\section{Statistical analysis}

All tests were carried out using intent-to-treat analysis. All tests were two-sided using a significance level of 0.05. Baseline comparisons between groups were performed using analysis of variance (ANOVA) and/or the chi-squared test, as appropriate. The primary outcome variable, the colonization of the oral cavity by target respiratory pathogens, was observed in each patient until their discharge from the ICU. A mixed effect model 
was used to compare overall treatment effects between groups for repeated measures data. It is known that, under the assumption that data is randomly missed, a likelihood based method (such as the mixed effect model) provides unbiased inference regardless of missing data. The group comparison for each time point was performed using ANOVA. All secondary continuous variables with repeated measures data were analyzed similarly. The group comparisons with categorical variables were carried out using the chi-squared test. Time to VAP was defined as the interval between the time of enrollment and time of the first diagnosis of pneumonia, as confirmed by a positive bqBAL. Probability plots for time to VAP were estimated by the Kaplan-Meier method. The distributions of time to VAP were compared using the Cox proportional hazards model.

\section{Results}

\section{Description of study participants}

The study design and patient recruitment outcomes are depicted in Figure 1. Initiation of patient recruitment commenced on 1 March, 2004 and ended on 30 November, 2007. A total of 2409 patients were admitted to the ICU during this period. Of these, a total of 820 were ventilated and therefore potentially eligible for inclusion. Patients were admitted to the study who showed no criteria for exclusion, and who, following consultation with the attending physician and nursing staff, would likely remain ventilated for at least 48 hours. Of the 175 patients who initially provided informed consent and who were randomly assigned to one of three groups (placebo - vehicle control, $\mathrm{CHX}$ once a day, $\mathrm{CHX}$ twice a day), 19 were extubated or expired before sampling. Another 10 subjects were eliminated from the analysis due to missing data, leaving 146 patients having microbiologic data at least at baseline. Thus, analysis of the primary outcomes (dental plaque score and the colonization of target pathogens present in dental plaque) was performed on data from 146 subjects. Of these, 115 were assessed at 48 hours (e.g. microbiologic assessment at the 48 hour time point ( $n=42$ in the placebo group, 36 in the $\mathrm{CHX}$ once a day group, and 37 in the $\mathrm{CHX}$ twice a day group), 93 subjects were assessed at 96 hours, 74 subjects at 144 hours, 69 at 192 hours, 57 subjects at 240 hours, 40 subjects at 288 hours, 30 subjects at 336 hours, 38 subjects at 384 hours, 20 subjects at 432 hours, and finally 12 subjects at 480 hours. In addition, intent to treat survival analysis was performed for all 175 patients who were initially randomized.

Demographic and clinical characteristics of the subjects enrolled in this study are presented in Table 1. The median age of the 146 study subjects was 48.0 years (range: 18.0 to 87.5), with the majority being white males admitted from the community. The groups were well balanced with respect to age, gender, APACHE score at baseline, and antibiotic exposure before inclusion in the study (Table 1). Of the 146 subjects, 16 were edentulous: 7 of 49 in the control group, 3 of 47 in the $\mathrm{CHX}$ once a day group, and 6 of 50 in the $\mathrm{CHX}$ twice a day group ( $P=0.4449)$. We found no correlations between co-morbidities and group assignment (Table 1).

\section{Dental plaque scores and microbiology results}

Neither $\mathrm{CHX}$ once a day or twice a day showed a significant reduction in plaque scores when compared with the placebo group (Figure 2, overall treatment effect $P=0.8423$ ). $\mathrm{CHX}$ delivered once or twice a day yielded similar results with respect to the number of PRPs in dental plaque samples. When compared with the placebo control group, no reduction in total counts or PRPs as a percentage of total cultivable flora were noted between the placebo and either $\mathrm{CHX}$ group, at all time points (Figure 3, overall treatment effect $P=0.6750$ ). In addition, the total number of cfus recovered as enterics, Pseudomonas and Acinetobacter were not reduced by $\mathrm{CHX}$ at any time point. A statistically significant reduction was, however, noted in the total number of $S$. aureus recovered in the $\mathrm{CHX}$ groups when compared with the placebo group (Figure 4, $P=$ 0.0065 and 0.0201 at day 2 and day 4 , respectively). $\mathrm{CHX}$ treatment also did not appear to reduce tracheal colonization by PRPs (data not shown).

Dental plaque samples from the first 100 subjects enrolled into the study were also plated on blood agar plates supplemented to $0.12 \% \mathrm{CHX}$. In all cases, these plates showed no growth, demonstrating that the intervention did not select for $\mathrm{CHX}$-resistant bacteria.

\section{Clinical pulmonary results}

No intra oral adverse events were noted, including mucositis or tooth staining. No significant differences were found between groups (Table 1) with respect to antibiotic use, ventilator days, days in the hospital, mean CPIS score (Figure 5), or mortality. When pneumonia was defined as the presence of more than $10^{4} \mathrm{cfu}$ of pathogen/ml of bqBAL fluid, among all 175 patients, 12 subjects in the control group were found to have pneumonia; in the $\mathrm{CHX}$ once a day group, 7 subjects were diagnosed with pneumonia, while in the $\mathrm{CHX}$ twice a day group 7 subjects also had pneumonia. Using intent-to-treat analysis, a $41 \%$ of reduction in the rate of pneumonia was noted between the treated and placebo group (odds ratio $(\mathrm{OR})=0.54,95 \%$ confidence interval $(\mathrm{Cl}): 0.23$ to $1.25, P=$ $0.1459)$; however, the differences were found not to be statistically significant $(P=0.1459)$. The incidence of pneumonia by survival analysis showed that the onset of pneumonia tended to be delayed in the treated groups when compared with the control group; however, these differences were not statistically significant (hazards ratio $(\mathrm{HR})=0.555,95 \% \mathrm{Cl}: 0.256$ to $1.201, P=0.1348$ ). If patients who were discharged or diagnosed with pneumonia on the day of enrollment into the study (five and one patients, respectively) were excluded, a decreased risk for VAP in the $\mathrm{CHX}$-treated group $(\mathrm{HR}=$ $0.514,95 \% \mathrm{Cl}: 0.234$ to $1.127, P=0.0966)$ was observed. 
Table 1

\begin{tabular}{|c|c|c|c|c|c|}
\hline Variables & $\begin{array}{l}\text { Total } \\
(n=146)\end{array}$ & $\begin{array}{l}\text { Control } \\
(n=49)\end{array}$ & $\begin{array}{l}\mathrm{CHX}^{\star} 1 \\
(\mathrm{n}=47)\end{array}$ & $\begin{array}{l}\mathrm{CHX}^{\star} 2 \\
(\mathrm{n}=50)\end{array}$ & $P$ value \\
\hline Age (years) & $48.0 \pm 20.8$ & $50.0 \pm 22.5$ & $44.8 \pm 19.9$ & $47.6 \pm 19.1$ & 0.3948 \\
\hline Gender - male/female & $104 / 42$ & $36 / 23$ & $43 / 15$ & $44 / 14$ & 0.1570 \\
\hline Race & & & & & 0.3661 \\
\hline White & $117(88 \%)$ & $43(88 \%)$ & $37(78 \%)$ & $37(74 \%)$ & \\
\hline African American & $24(17 \%)$ & $4(8 \%)$ & $10(22 \%)$ & $10(20 \%)$ & \\
\hline Asian & $2(1 \%)$ & $1(2 \%)$ & $0(0 \%)$ & $1(2 \%)$ & \\
\hline American Indian & $3(1 \%)$ & $1(2 \%)$ & $0(0 \%)$ & $2(4 \%)$ & \\
\hline Admitted from & & & & & 0.0989 \\
\hline Internal hospital ward & $6(4 \%)$ & $5(10 \%)$ & $1(25)$ & $0(0 \%)$ & \\
\hline External community & $116(80 \%)$ & $38(76 \%)$ & $37(79 \%)$ & $41(82 \%)$ & \\
\hline Other & $24(16 \%)$ & $6(12 \%)$ & $9(19 \%)$ & $9(18 \%)$ & \\
\hline Co-morbidities & $68(47 \%)^{*}$ & $24(50 \%)$ & $19(40 \%)$ & $25(51 \%)$ & 0.5212 \\
\hline Cardiac & $35(24 \%)$ & $13(27 \%)$ & $10(21 \%)$ & $12(25 \%)$ & 0.8039 \\
\hline Pulmonary & $16(11 \%)$ & $7(15 \%)$ & $3(6 \%)$ & $6(12 \%)$ & 0.4245 \\
\hline Renal & $5(3 \%)$ & $2(4 \%)$ & $0(0 \%)$ & $3(6 \%)$ & 0.2483 \\
\hline Hepatic & $2(1 \%)$ & $2(4 \%)$ & $0(0 \%)$ & $0(0 \%)$ & 0.1316 \\
\hline Endocrine & $24(17 \%)$ & $10(21 \%)$ & $6(13 \%)$ & $8(16 \%)$ & 0.5715 \\
\hline Central nervous & $21(15 \%)$ & $9(19 \%)$ & $8(17 \%)$ & $4(8 \%)$ & 0.2844 \\
\hline Neoplastic & $10(7 \%)$ & $4(8 \%)$ & $2(4 \%)$ & $4(8 \%)$ & 0.6764 \\
\hline Immunosuppression & $2(1 \%)$ & $0(0 \%)$ & $0(0 \%)$ & $2(4 \%)$ & 0.1400 \\
\hline APACHE (baseline) & $19.1 \pm 5.5$ & $19.1 \pm 6.1$ & $18.5 \pm 4.1$ & $19.7 \pm 6.1$ & 0.5850 \\
\hline Antibiotic exposure before study inclusion & $43(29 \%)$ & $18(37 \%)$ & $12(26 \%)$ & $13(26 \%)$ & 0.3896 \\
\hline Antibiotic exposure after study inclusion & $103(71 \%)$ & $32(65 \%)$ & $34(72 \%)$ & $37(74 \%)$ & 0.6043 \\
\hline Antibiotic use & $3.5 \pm 3.6$ & $3.1 \pm 3.5$ & $3.8 \pm 3.6$ & $3.7 \pm 3.8$ & 0.6057 \\
\hline Days ventilated & $9.1 \pm 5.6$ & $9.7 \pm 6.3$ & $9.4 \pm 5.0$ & $8.4 \pm 5.2$ & 0.5180 \\
\hline Days in hospital & $11.4 \pm 6.6$ & $11.3 \pm 6.7$ & $12.0 \pm 6.3$ & $11.0 \pm 6.8$ & 0.7263 \\
\hline Deaths & $24(17 \%)$ & $8(17 \%)$ & $8(17 \%)$ & $8(16 \%)$ & 0.9834 \\
\hline Missing doses & $0.7 \pm 1.1$ & $0.9 \pm 1.2$ & $0.7 \pm 1.3$ & $0.5 \pm 0.8$ & 0.2844 \\
\hline
\end{tabular}

*Some patients had multi-comorbidities.

APACHE = Acute Physiology and Chronic Health Evaluation; $\mathrm{CHX}=$ chlorhexidine gluconate.

No differences were noted between groups in the number of missed doses of $\mathrm{CHX}$ (or placebo) delivered to each subject (Table 1).

\section{Discussion}

As VAP continues to be a common complication of critical care, development of preventive approaches are essential to reduce the incidence of this costly infection. The goal of the present trial was to determine the minimum frequency (once or twice a day) of oral decontamination with $0.12 \mathrm{CHX}$ required to improve oral hygiene and reduce oral colonization by potential respiratory pathogens in intubated MV patients admitted to the trauma ICU. The strengths of this trial include the well-controlled, randomized, blinded, well-concealed, and blocked design, and the fact that the intervention was provided by staff nurses, thus allowing for the test of the intervention under 'real world' conditions.

The results show that the use of oral topical $\mathrm{CHX}$ resulted in a quantitative reduction in the number of $S$. aureus cfus in the 


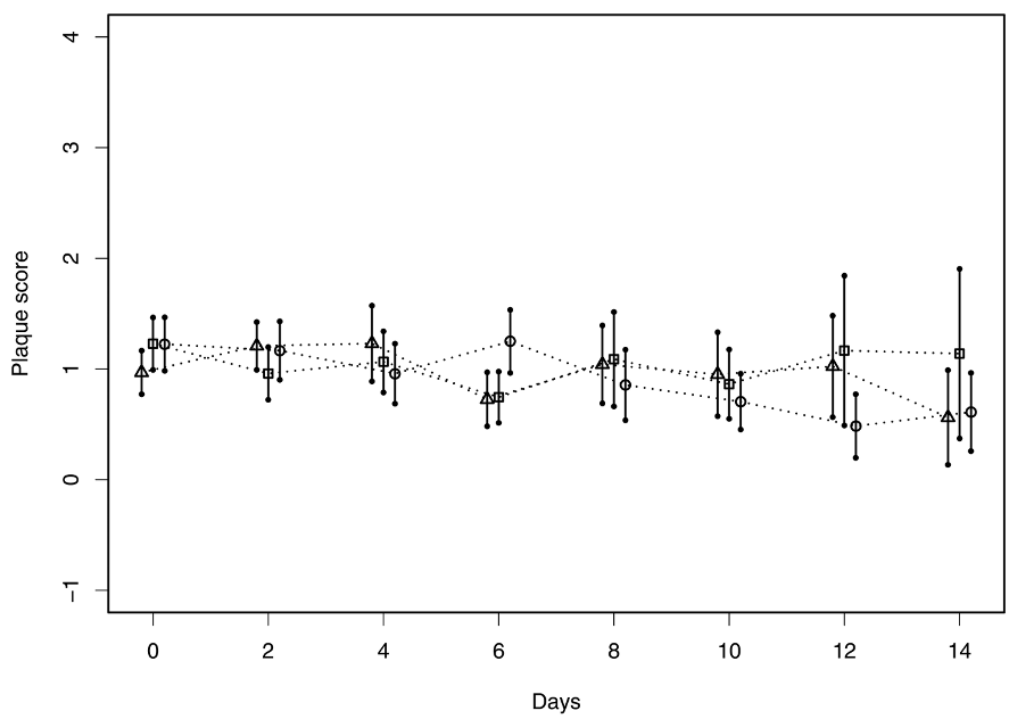

Effect of topical $\mathrm{CHX}$ on dental plaque scores (mean) and 95\% confidence intervals. Triangle = placebo; Square = chlorhexidine gluconate $(\mathrm{CHX})$ once a day; Circle $=\mathrm{CHX}$ twice a day. Days represent duration following mechanical ventilation. The confidence intervals were obtained based on data at each time point.

dental plaque of MV-ICU patients. The finding that $\mathrm{CHX}$ treatment reduced the number of $S$. aureus in the oral cavity supports previously published studies that suggest that $S$. aureus is vulnerable to $\mathrm{CHX}$ disinfection [14]. However, $\mathrm{CHX}$ did not appear to reduce the total number or proportion of other target PRPs in dental plaque (Pseudomonas, Acinetobacter or enteric species). Although the group size was not sufficiently large to show a statistically significant reduction in the inci- dence of VAP between groups, there was a trend for fewer cases of VAP in both $\mathrm{CHX}$ groups.

There are several possible explanations to explain why $\mathrm{CHX}$ did not reduce the numbers of Gram-negative pathogens in oral biofilms. First, it is known that Gram-positive pathogens such as $\mathrm{S}$. aureus are more sensitive to $\mathrm{CHX}$ than are Gramnegative pathogens $[13,24]$. Second, because dental plaque biofilm was the primary oral sample analyzed, it is likely that the

Figure 3

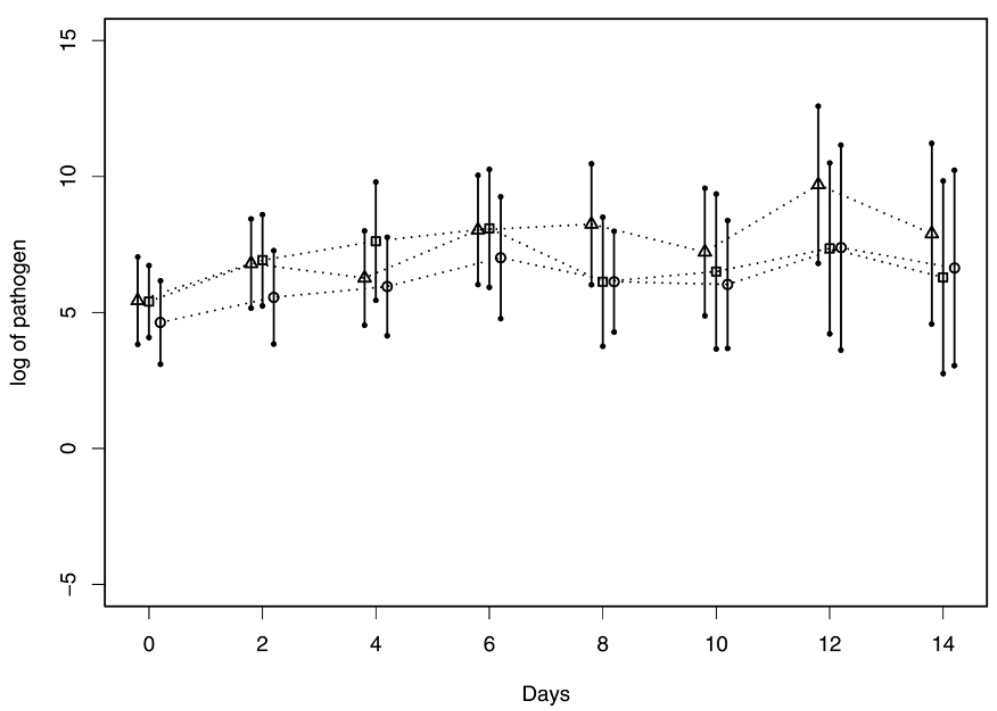

Effect of topical chlorhexidine gluconate on the number of potential respiratory bacterial pathogens in oral samples. Mean in log scale with $95 \%$ confidence intervals. The confidence intervals were obtained based on data at each time point. Triangle = placebo; Square = chlorhexidine gluconate $(\mathrm{CHX})$ once a day; Circle $=\mathrm{CHX}$ twice a day. 


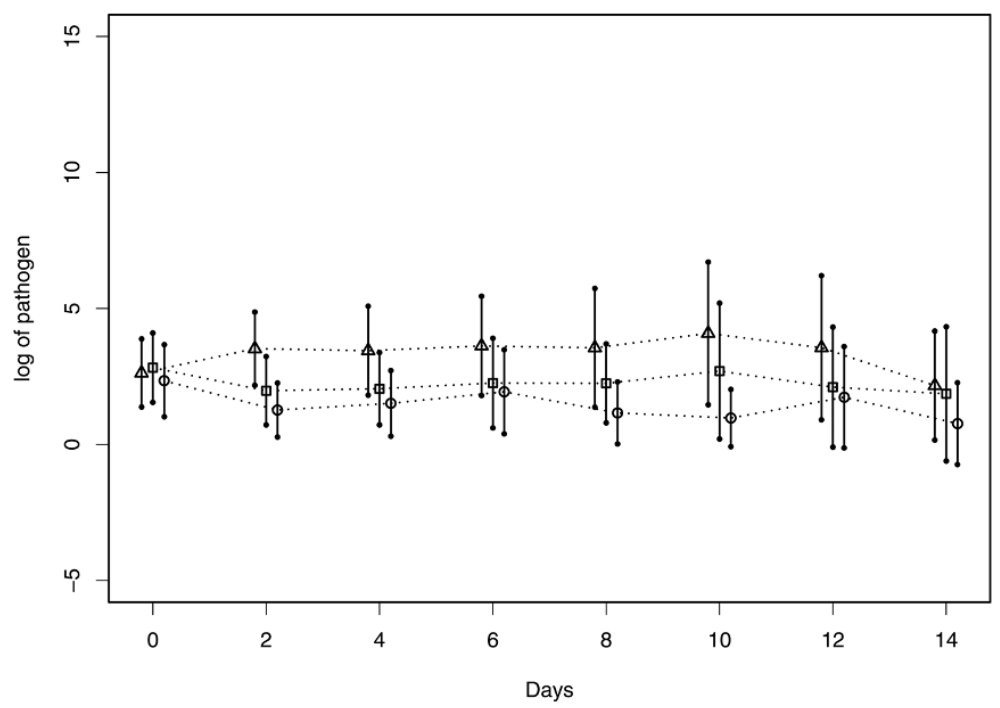

Effect of topical chlorhexidine gluconate on the number of Staphylococcus aureus in oral samples. Mean in log scale with $95 \%$ confidence intervals. The confidence intervals were obtained based on data at each time point. Triangle $=$ placebo; Square $=$ chlorhexidine gluconate $(\mathrm{CHX})$ once a day; Circle $=\mathrm{CHX}$ twice a day.

organisms cultured from the biofilm were resistant to the topically delivered antimicrobial agent. It is well known that bacteria that adopt a biofilm lifestyle are usually resistant to antimicrobial agents. Such resistance has been attributed to slow growth in the biofilm, the induction of stress responses, and or production of exopolysaccharides or other extracellular components that exclude the antimicrobial agent from the bacterial community within the biofilm [25].
A number of published studies suggest that topical $\mathrm{CHX}$ twice a day prevents VAP [11-15,26]. Furthermore, several studies have demonstrated the genetic similarity of bacteria isolated from the lung to bacteria isolated from dental plaque, demonstrating that dental biofilms are an important reservoir for these pathogens $[4,5]$. Thus, mechanisms other than reduction of PRPs in dental biofilm must be considered to help explain the apparent efficacy of this agent to prevent VAP. One possibility is that $\mathrm{CHX}$ inhibits the viability of the planktonic bacteria in the

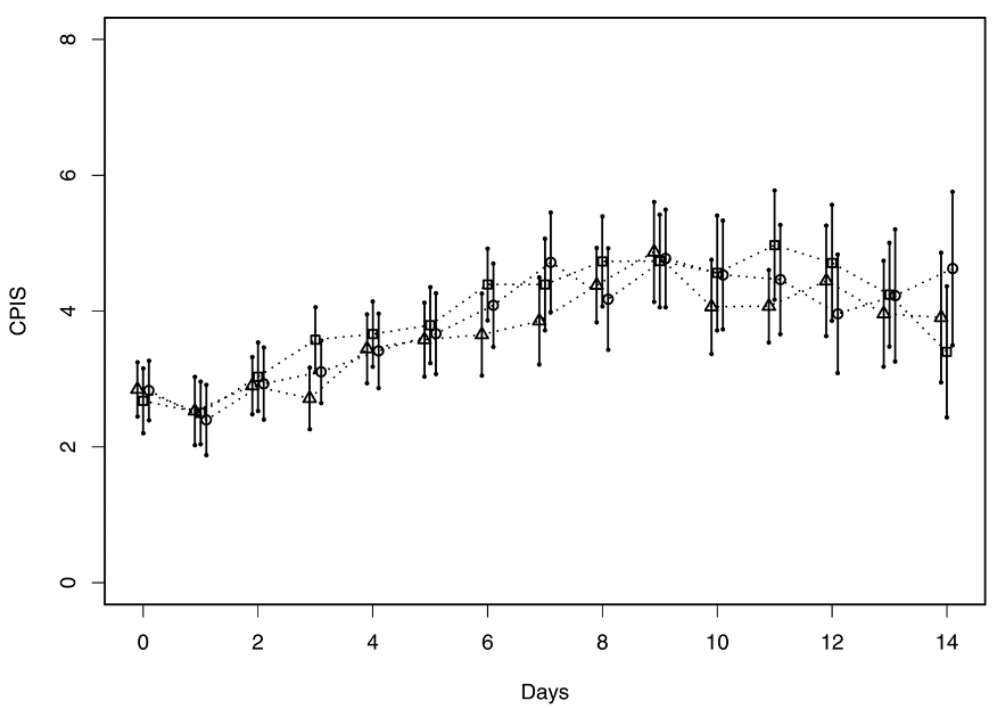

Effect of $\mathrm{CHX}$ on mean clinical pulmonary infection score with $95 \%$ confidence intervals. Triangle = placebo; Square = chlorhexidine gluconate $(\mathrm{CHX})$ once a day; Circle $=\mathrm{CHX}$ twice a day. The confidence intervals were obtained based on data at each time point. 
oral secretions. The subsequent reduction in the number of viable PRPs in the secretions thus reduces the number of viable organisms aspirated into the lower airway and therefore will prevent subsequent infection. Alternatively, the virulence potential of the bacteria may be reduced by $\mathrm{CHX}$. Previous studies have suggested that $\mathrm{CHX}$ is able to bind to bacterial components such as lipopolysaccharide and proteases [2729]. Such interactions may diminish the biologic activity of such components to reduce the virulence potential of bacteria. It is also possible that concomitant use of other oral care products such as toothpaste might reduce $\mathrm{CHX}$ efficacy [30].

Adverse events have rarely been reported in clinical trials of $\mathrm{CHX}$ in ICU patients. A meta-analysis of seven clinical trials found that adverse effects were not reported in any of these studies [31]. A recent clinical trial of $2 \% \mathrm{CHX}$ versus saline found that $9.8 \%$ in the $\mathrm{CHX}$ group had mucosal irritation versus $0.9 \%$ in saline controls $(P=.001)$. The present study using $0.12 \% \mathrm{CHX}$ found no adverse effects (mucosal irritation or tooth staining).

No previous published clinical trials of $\mathrm{CHX}$ in the context of VAP have assessed resistance to this agent during the study. In the present study, an effort was made to identify resistance to $\mathrm{CHX}$ by plating samples of dental plaque or lower airway secretions on agar containing $0.12 \% \mathrm{CHX}$. Although this approach most likely detects high level $\mathrm{CHX}$ resistance, no such resistant bacterial colonies were noted using this method. This finding indicates that resistance to $\mathrm{CHX}$ was not likely to be an explanation for the findings of this study.

Previous meta-analyses of trials conclude that this $\mathrm{CHX}$ is effective in prevention of VAP [31,32]. These analyses revealed, however, that there was variation in the populations studied as well as in the concentration, preparation, and dosing schedule of $\mathrm{CHX}$. Clinical trials of $\mathrm{CHX}$ have tested concentrations of $0.12 \%, 0.2 \%$, and $2 \%$, applied two to four times a day, and delivered as a rinse, gel or in Vaseline. Thus, although topical application of $\mathrm{CHX}$ to the oral cavity of ventilated ICU patients in some cases appears to prevent VAP, the optimal concentration and frequency of application of this agent has not been validated. The present study was designed to determine if there was any difference in oral colonization with PRPs between once versus twice daily $0.12 \% \mathrm{CHX}$. There was no significant difference between once versus twice daily $0.12 \% \mathrm{CHX}$ solution in terms of reducing oral colonization by PRPs, with the exception of $S$. aureus, whose numbers were reduced in dental plaque of individuals treated with $\mathrm{CHX}$ delivered once or twice a day.

Other environmental factors may also help explain the apparent limitation of $\mathrm{CHX}$ to reduce the number of PRPs in the oral cavity. The nurses at the hospital where the trial was conducted are well educated on the possible role of oral hygiene in VAP. The standardized oral care regime in place includes the debridement of the teeth and tongue by use of a suction toothbrush used twice a day together with swabbing with a peroxide rinse, swabbing with a peroxide rinse-impregnated swab every four hours, and use of a mouth moisturizer applied to the oral mucosa. Deep oropharyngeal suctioning is performed prior to major position changes and extubation, and as needed to remove oropharyngeal secretions that have pooled on top of the cuff of the endotracheal tube. This regime may have already reduced the number of organisms in the dental plaque to a level where additional reductions by $\mathrm{CHX}$ were not detectable. On the other hand, suctioning excess fluid at the time of application could have reduced the effect of $\mathrm{CHX}$. Also, approximately $70 \%$ of the subjects enrolled in the present study were given antibiotics for reasons other than the development of VAP during the course of their ICU stay, for example for surgical prophylaxis or for treatment of another infection. Such exposure to antibiotics might have reduced the efficacy of $\mathrm{CHX}$ in this setting.

In summary, $\mathrm{CHX}$ applied topically once or twice a day to the oral cavity inhibited the numbers of $S$. aureus in the dental plaque of MV-ICU patients. An absence of an effect on the total number of target pathogens, or on Gram-negative species, is consistent with previous studies [13]. Perhaps an increase in the concentration of $\mathrm{CHX}$ or in the frequency of application would be more effective in reduction of pathogenic bacteria in oral biofilms. Additional large-scale clinical trials are required to determine the efficacy of $\mathrm{CHX}$ in the prevention of VAP in MV-ICU patients. Alternative approaches to reduce or eliminate PRPs from the oral cavity should also be studied, which may include other topical chemotherapeutic agents or frequent mechanical dislodgment of biofilms from the oral surfaces.

\section{Conclusions}

The results of this trial show that oral topical $\mathrm{CHX}$ reduces the number of viable $S$. aureus in the dental plaque of MV-ICU patients. However, $\mathrm{CHX}$ did not reduce the total number or proportion of other target PRPs (Pseudomonas, Acinetobacter or enteric species) in dental plaque.

\section{Competing interests}

The authors declare that they have no competing interests.

\section{Authors' contributions}

FAS conceived, designed and supervised the study, participated in analysis and interpretation of the data, drafted the manuscript. JY statistically analyzed that data and contributed to its interpretation. KR monitored subject inclusion and critically reviewed and revised the manuscript. JMM contributed to the study design, data interpretation and critically reviewed and revised the manuscript. AV acquired the data. SIO and $\mathrm{KW}$ organized and managed the data. All authors read and approved the final manuscript. 


\section{Key messages}

- Oral topical $\mathrm{CHX}$ reduces the number of viable $S$. aureus in the dental plaque of MV-ICU patients.

- $\mathrm{CHX}$ did not reduce the total number or proportion of other target PRPs in dental plaque, such as Pseudomonas, Acinetobacter or enteric species.

- No intra oral adverse events were noted, including mucositis or tooth staining.

- Although $\mathrm{CHX}$ reduced the number of cases of VAP compared with the placebo, the differences were found not to be statistically significant.

- No differences were noted for any outcome measured between groups treated once or twice a day with $\mathrm{CHX}$.

\section{Acknowledgements}

We thank Noreen Frawley, R.N. for nursing assistance; Dr. Daniel Amsterdam of the ECMC laboratory for facilitation of the microbiology studies; Paul Stockdill of the ECMC pharmacy for preparation of the $\mathrm{CHX}$ and placebo rinses; Gregory Pavlov for facilitation of data management; and Dr Alan Hutson, for statistical consultation during the trial planning. This study was supported by USPH grant R01DE-14685 from the National Institute of Dental and Craniofacial Research.

\section{References}

1. Verghese A, Berk SL: Bacterial pneumonia in the elderly. Medicine (Baltimore) 1983, 62:271-285.

2. Scannapieco FA, Stewart EM, Mylotte JM: Colonization of dental plaque by respiratory pathogens in medical intensive care patients. Crit Care Med 1992, 20:740-745.

3. Fourrier F, Duvivier B, Boutigny H, Roussel-Delvallez M, Chopin C: Colonization of dental plaque: a source of nosocomial infections in intensive care unit patients. Crit Care Med 1998, 26:301-308.

4. El-Solh AA, Pietrantoni C, Bhat A, Okada M, Zambon J, Aquilina A, Berbary $E$ : Colonization of dental plaques: a reservoir of respiratory pathogens for hospital-acquired pneumonia in institutionalized elders. Chest 2004, 126:1575-1582.

5. Heo SM, Haase EM, Lesse AJ, Gill SR, Scannapieco FA: Genetic relationships between respiratory pathogens isolated from dental plaque and bronchoalveolar lavage fluid from patients in the intensive care unit undergoing mechanical ventilation. Clin Infect Dis 2008, 47:1562-1570.

6. Tonelli PM, Hume WR, Kenney EB: Chlorhexidine: a review of the literature. J West Soc Periodontol Periodontal Abstr 1983, 31:5-10.

7. Addy M: Chlorhexidine compared with other locally delivered antimicrobials. A short review. J Clin Periodontol 1986, 13:957-964.

8. Lang NP, Brecx MC: Chlorhexidine gluconate-an agent for chemical plaque control and prevention of gingival inflammation. J Periodont Res 1986, 21(Suppl 16):74-89.

9. DeRiso AJ 2nd, Ladowski JS, Dillon TA, Justice JW, Peterson AC: Chlorhexidine gluconate $0.12 \%$ oral rinse reduces the incidence of total nosocomial respiratory infection and nonprophylactic systemic antibiotic use in patients undergoing heart surgery. Chest 1996, 109:1556-1561.

10. Fourrier $F$, Cau-Pottier $E$, Boutigny $H$, Roussel-Delvallez $M$, Jourdain $M$, Chopin C: Effects of dental plaque antiseptic decontamination on bacterial colonization and nosocomial infections in critically ill patients. Intensive Care Med 2000, 26:1239-1247.

11. Genuit T, Bochicchio G, Napolitano LM, McCarter RJ, Roghman MC: Prophylactic chlorhexidine oral rinse decreases ventilator-associated pneumonia in surgical ICU patients. Surg Infect (Larchmt) 2001, 2:5-18.
12. Houston S, Hougland $P$, Anderson JJ, LaRocco M, Kennedy V, Gentry LO: Effectiveness of $0.12 \%$ chlorhexidine gluconate oral rinse in reducing prevalence of nosocomial pneumonia in patients undergoing heart surgery. Am J Crit Care 2002, 11:567-570.

13. Koeman $M$, Ven AJ van der, Hak E, Joore HC, Kaasjager $K$, de Smet AG, Ramsay G, Dormans TP, Aarts LP, de Bel EE, Hustinx WN, Tweel I van der, Hoepelman AM, Bonten MJ: Oral decontamination with chlorhexidine reduces the incidence of ventilatorassociated pneumonia. Am J Respir Crit Care Med 2006, 173(12):1348-1355.

14. Segers $P$, Speekenbrink RG, Ubbink DT, van Ogtrop ML, de Mol BA: Prevention of nosocomial infection in cardiac surgery by decontamination of the nasopharynx and oropharynx with chlorhexidine gluconate: a randomized controlled trial. JAMA 2006, 296:2460-2466.

15. Lansford T, Moncure M, Carlton E, Endress R, Shik N, Udobi K, Braxton C, Danks R: Efficacy of a pneumonia prevention proto$\mathrm{col}$ in the reduction of ventilator-associated pneumonia in trauma patients. Surgical Infections 2007, 8:505-510.

16. Mori $H$, Hirasawa $H$, Oda $S$, Shiga $H$, Matsuda K, Nakamura M: Oral care reduces incidence of ventilator-associated pneumonia in ICU populations. Intensive Care Med 2006, 32:230-236.

17. Tablan OC, Anderson LJ, Besser R, Bridges C, Hajjeh R: Guidelines for preventing health-care - associated pneumonia, 2003: recommendations of CDC and the Healthcare Infection Control Practices Advisory Committee. MMWR Recomm Rep 2004, 53:1-36.

18. Craven DE: Preventing ventilator-associated pneumonia in adults: sowing seeds of change. Chest 2006, 130:251-260.

19. Pineda LA, Saliba RG, El Solh AA: Effect of oral decontamination with chlorhexidine on the incidence of nosocomial pneumonia: a meta-analysis. Crit Care 2006, 10:R35.

20. Drug information for the health care professional. Englewood, CO: Micromedex, Inc; 2000.

21. Pugin J, Auckenthaler R, Mili N, Janssens JP, Lew PD, Suter PM: Diagnosis of ventilator-associated pneumonia by bacteriologic analysis of bronchoscopic and nonbronchoscopic "blind" bronchoalveolar lavage fluid. Am Rev Respir Dis 1991, 143:1121-1129.

22. Kollef $\mathrm{MH}$, Ward $\mathrm{S}$ : The influence of mini-BAL cultures on patient outcomes: implications for the antibiotic management of ventilator-associated pneumonia. Chest 1998, 113:412-420.

23. Knaus WA, Draper EA, Wagner DP, Zimmerman JE: APACHE II: A severity of disease classification system. Crit Care Med 1985, 13:818-829.

24. Block C, Furman M: Association between intensity of chlorhexidine use and micro-organisms of reduced susceptibility in a hospital environment. J Hosp Infect 2002, 51:201-206.

25. Mah TF, O'Toole GA: Mechanisms of biofilm resistance to antimicrobial agents. Trends Microbiol 2001, 9:34-39.

26. Fourrier $F$, Dubois $D$, Pronnier $P$, Herbecq $P$, Leroy $O$, Desmettre T, Pottier-Cau E, Boutigny H, Di Pompeo C, Durocher A, RousselDelvallez M, PIRAD Study Group: Effect of gingival and dental plaque antiseptic decontamination on nosocomial infections acquired in the intensive care unit: a double-blind placebocontrolled multicenter study. Crit Care Med 2005, 33:1728-1735.

27. Grenier D, Bertrand J, Mayrand D: Porphyromonas gingivalis outer membrane vesicles promote bacterial resistance to chlorhexidine. Oral Microbiol Immunol 1995, 10:319-320.

28. Gendron R, Grenier D, Sorsa T, Mayrand D: Inhibition of the activities of matrix metalloproteinases 2,8 , and 9 by chlorhexidine. Clin Diagn Lab Immunol 1999, 6:437-439.

29. Cronan CA, Potempa J, Travis J, Mayo JA: Inhibition of Porphyromonas gingivalis proteinases (gingipains) by chlorhexidine: synergistic effect of $\mathrm{Zn}(\mathrm{II})$. Oral Microbiol Immunol 2006, 21:212-217.

30. Kolahi J, Soolari A: Rinsing with chlorhexidine gluconate solution after brushing and flossing teeth: a systematic review of effectiveness. Quintessence Int 2006, 37:605-612.

31. Chlebicki MP, Safdar N: Topical chlorhexidine for prevention of ventilator-associated pneumonia: a meta-analysis. Crit Care Med 2007, 35:595-602. 
Critical Care Vol 13 No 4 Scannapieco et al.

32. Chan EY, Ruest A, Meade MO, Cook DJ: Oral decontamination for prevention of pneumonia in mechanically ventilated adults: systematic review and meta-analysis. BMJ 2007, 334:889. 